\section{Desafíos para el Caribe a 10 años de la Conferencia Internacional sobre la Población y el Desarrollo de El Cairo, Egipto}

\author{
Leticia Artiles Visbal ${ }^{1}$
}

Palabras clave: formulación de políticas, derechos de la mujer, Región del Caribe, salud reproductiva, género, sexualidad.

\footnotetext{
1 Agencia de Cooperación para la Movilización de Recursos, Instituto Superior de Ciencias Médicas de La Habana. Dirección postal: Edificio Ramón Paz Borroto, calle G esquina a 25, El Vedado, Ciudad de La Habana, Cuba. Correo electrónico: leticia@infomed.sld.cu

2 Aunque fue en la Conferencia Internacional de Derechos Humanos, celebrada en Teherán, Irán, en 1968, donde se habló por primera vez del derecho humano a determinar libremente el número de hijos y el intervalo entre sus nacimientos, no fue hasta finales del siglo pasado, en la Conferencia Internacional sobre la Población y el Desarrollo, celebrada en El Cairo, Egipto, en 1994, que se acuñó el termino "derechos reproductivos" para designar el conjunto de derechos humanos relacionados con la salud reproductiva y, más ampliamente, todos los derechos humanos que inciden sobre la reproducción humana, incluidos los vinculados al binomio población-desarrollo sostenible. Facio A. Asegurando el futuro: las instituciones nacionales de derechos humanos y los derechos reproductivos. Conferencia impartida en el seminario del Fondo de Población de las Naciones Unidas (UNFPA). Metodología con Enfoque de Género, los Derechos Humanos y los Derechos Sexuales y Reproductivos, Caracas, Venezuela, 11 de junio de 2003.
}

En los albores del siglo XXI, fuerzas conservadoras expresaron abiertamente en algunos países su oposición a los acuerdos de la Conferencia Internacional sobre la Población y el Desarrollo (CIPD) que se celebró en El Cairo, Egipto, en 1994, con el argumento de que el Programa de Acción aprobado en ella - conocido también como "Agenda Cairo" - fomenta la actividad sexual en menores de edad y el aborto (1). Ello refleja la realidad de que, a estas alturas, en el camino de los derechos sexuales y reproductivos "queda mucho por andar".

En la CIPD, celebrada bajo el auspicio de las Naciones Unidas, participaron representantes de 179 países. En esa reunión se lograron avances importantes: se reconoció la estrecha relación que existe entre los conceptos de población, crecimiento económico y desarrollo sostenible; se reafirmó el nexo entre la población, el desarrollo y la educación, y entre la tecnología, la investigación y el desarrollo; y se reconoció la relación existente entre las migraciones y la salud. Sin embargo, los avances más significativos fueron los referidos a la salud sexual y a la salud reproductiva, pues se reconocieron los derechos reproductivos como parte de los derechos humanos, ${ }^{2}$ se promovieron sostenidamente la igualdad y la equidad entre los sexos y la habilitación de la mujer, y se reconoció a la familia como la célula básica de la sociedad donde cotidianamente se transmite la cultura. En este contexto se introdujeron o reafirmaron importantes conceptos, entre ellos los de planificación familiar, salud sexual, salud reproductiva, derechos reproductivos y maternidad sin riesgo (2). Por su parte, el Programa de Acción aprobado se acogió como una plataforma para el diseño de políticas nacionales e internacionales en materia de población y desarrollo para un período de 20 años.

Este Programa de Acción fue la vía para implementar los acuerdos de la CIPD y sus resultados se evalúan cada cinco años a partir de su aprobación. En estos análisis evaluativos se han reconocido los importantes avances obtenidos en la lucha contra la pobreza, en materia de derechos humanos, en el mejoramiento de las condiciones de vida de grupos sociales y étnicos desfavorecidos, en la adopción de nuevas leyes y normativas, y en la implementación de programas de acción en los Estados signatarios.

El primer examen quinquenal de los avances logrados se realizó en Nueva York en 1999 durante el 21..$^{\circ}$ período extraordinario de sesiones de la Asamblea General de las Naciones Unidas. En esa reunión se constató la necesidad de eliminar los obstáculos de todo tipo - ya fueran financieros, institucionales 
CUADRO 1. Algunos indicadores relacionados con la salud reproductiva en el Caribe

\begin{tabular}{lrr}
\hline \multicolumn{1}{c}{ Indicador } & Caribe latino $^{\mathrm{a}}$ & Caribe no latino $^{\mathrm{b}}$ \\
\hline Atención del embarazo por personal técnicamente capacitado, \% & 89,7 & 96,0 \\
Uso de anticonceptivos, \% & 71 & 53 \\
Tasa de mortalidad materna, por 100 000 nacidos vivos & 73 & 239 \\
Tasa de mortalidad perinatal, por 1 000 nacidos vivos & 54 & 32 \\
Tasa de mortalidad Infantil, por 1 000 nacidos vivos & 41,3 & 19,3 \\
\hline
\end{tabular}

Fuente: Centro Latinoamericano de Perinatología y Desarrollo Humano (3).

a El Caribe latino está constituido por Cuba, Haití, Puerto Rico y República Dominicana. Tiene una superficie total de $196242 \mathrm{~km}^{2}$ y 31791204 habitantes.

${ }^{\mathrm{b}}$ El Caribe no latino está constituido por 24 países y territorios. Tiene una superficie total de $505832 \mathrm{~km}^{2}$ y 8093312 habitantes.

o humanos- que impedían alcanzar los objetivos del Programa de Acción acordado en El Cairo.

Otro obstáculo importante aún presente es la natural resistencia humana a los cambios culturales, a asimilar un nuevo marco conceptual con respecto a la salud sexual y la salud reproductiva, y los cambios conductuales correspondientes, sobre todo en el escenario etnocentrista, patriarcal y conservador que predomina en muchos de los países que en 1994 aprobaron los acuerdos de El Cairo y el Programa de Acción. Esto se reflejó en la relatoría de la Reunión de Redes y Campañas Regionales para América Latina y el Caribe, celebrada en La Paz, Bolivia, en agosto de 2003, para conciliar las estrategias de los países de la Región de las Américas en este sentido. En esta reunión quedó evidenciado que, a pesar de los importantes avances logrados cinco años después de la reunión de El Cairo, el cumplimiento de las agendas consensuadas en los años 1994 y 1995 estaba en peligro. Los participantes consideraron que algunas medidas adoptadas por los gobiernos de algunos países en los últimos años influían negativamente en la implementación del Programa de Acción de El Cairo y afectaban al disfrute real de los derechos sexuales y reproductivos. Por otra parte, algunos países que apoyaban políticas pronatalistas y conservadoras también pretenden influir en el consenso de las acciones que se adopten en 2004 para el cumplimiento de las metas de la CIPD. ${ }^{3}$

\section{La situación de la Región del Caribe}

En la Región del Caribe se pueden diferenciar el llamado Caribe latino (CL), compuesto por Cuba, Haití, Puerto Rico y República Dominicana, y el Caribe no latino (CNL), compuesto por 24 países y territorios (cuadro 1 ).

\footnotetext{
3 Relatoría. Reunión de Redes y Campañas Regionales Cairo + 10. La Paz, Bolivia, 30 y 31 de agosto de 2003.
}

Desde el punto de vista antropológico, las naciones del Caribe conforman un complejo mosaico por su gran diversidad geográfica, étnica, lingüística, religiosa y de clases sociales, así como por la estructura institucional y funcional de sus sistemas de gobierno. Desde el punto de vista epidemiológico resultan sustantivas las diferencias existentes entre los diversos países en cuanto a las principales causas de muerte y la prevalencia de enfermedades prevenibles; algunos países presentan indicadores similares a los de los países industrializados y otros marcados por la extrema pobreza. Aun dentro de un mismo país, existen diferencias notables entre grupos poblacionales diferentes.

Esta mezcla determina diferencias en los perfiles epidemiológicos según el territorio social, la clase, la etnia y el género, y exige que todo lo relacionado con el binomio población-desarrollo, en particular lo referido a los derechos reproductivos y de la salud sexual y la salud reproductiva, sea tratado de manera holística. Esta situación se hace más compleja debido a que el ámbito de la salud sexual y reproductiva es, por sí mismo, una urdimbre de complejas relaciones por la concordancia de esas dos categorías y su expresión particular y diferencial en las distintas etapas del ciclo de vida, por los mitos y tabúes, por las jerarquías de poder etnocéntricas, patriarcales y mecanicistas, y por la diversidad en la orientación sexual que generan diferentes necesidades sociales. La complejidad de este contexto antropológico, geográfico y cultural requiere el diseño de estrategias y respuestas que acepten conscientemente la diversidad con un ordenamiento sistémico e integral adecuado a las peculiaridades de la Región.

Muchos países caribeños tienen una visión muy particular de los temas que forman parte de sus agendas de acción en respuesta a los acuerdos de la CIPD. En muchos de los países de esta subregión, el género - como categoría general transversalizadora de clase, etnia y territorio- se asume acríticamente $y$, aunque queda refrendada en discursos y políticas, se limita al tema de la mujer, particularmente a la ecuación mujer $=$ madre . Las nece- 
sidades sociales de orientación y educación sobre temas relacionados con la sexualidad aún no están satisfechas y los servicios de planificación familiar no son suficientes, pues cuando existen delegan la responsabilidad de la conducta de anticoncepción casi exclusivamente en la mujer.

El tema de la violencia doméstica aparece en el discurso oficial con el reclamo de que sea parte de las políticas nacionales. Aunque se realizan investigaciones, se crean redes y se toman decisiones, todavía las pocas acciones emprendidas quedan circunscritas a experiencias puntuales, como las llamadas casas de recogidas y las líneas telefónicas de ayuda, que no logran la transversalidad sectorial y el alcance suficientes para dar respuesta a las necesidades de las mujeres que sufren la violencia. En este sentido, las acciones emprendidas de carácter institucional, jurídico, policial e, incluso, de salud pública resultan insuficientes para lograr que la violencia contra la mujer y los niños deje de considerarse un "problema privado" y se convierta de hecho y derecho en un problema público (3).

El Caribe no ha quedado excluido del proceso de reforma estructural que ha marcado a toda la Región de las Américas. Estos cambios pudieran parecer neutrales en materia de género, pero ocultan importantes sesgos que se manifiestan en la desvalorización del trabajo de las mujeres y en la falta de reconocimiento de la contribución económica que representa su trabajo no remunerado dentro del hogar. Como consecuencia, formulaciones aparentemente neutras - como la reducción de costos, el aumento de la eficacia y la eficiencia y la descentralizaciónimplican la transferencia de determinados costos de la economía remunerada a la economía doméstica basada en el trabajo no remunerado de las mujeres. Centroamérica y el Caribe se presentan como un escenario de ajustes macroeconómicos con reformas institucionales en diferentes etapas de implantación que no contemplan la equidad de género ni la justicia social (4). Se debe recordar que estos países están marcados por grandes inequidades sociales, de etnia y de clase. La inequidad de género constituye un árbol más en ese bosque de desigualdades.

La alta prevalencia de cánceres ginecológicos, la feminización de la epidemia del sida y de la infección por VIH, el embarazo en adolescentes, el mercado y el tráfico sexual de mujeres, niñas y niños, y la exclusión de los inmigrantes por su propia condición o por su origen étnico, clase o color de la piel son una realidad en los países y territorios caribeños. Esta situación está en total contradicción con los acuerdos adoptados y asumidos por estos países durante la CIPD.

Existen dificultades para lograr un enfoque de género en el tratamiento de la información de salud - particularmente de la salud sexual y la salud reproductiva - que puedan ayudar a formular políticas nacionales. No existen registros estadísti- cos y cuando existen no son confiables o no están desagregados por sexo; la mala calidad del registro de la información no permite diseñar indicadores que definan claramente lo que se quiere medir. Además, se necesita diseñar indicadores de género que ayuden a planificar los presupuestos financieros, que sirvan como plataforma para implantar políticas y que permitan desmitificar los indicadores que miden aspectos relacionados con la salud reproductiva y los "problemas de la mujer". La eliminación de las manifestaciones de inequidad de género en el sistema de estadísticas de salud permitirá sustentar las bases teóricas y el diseño de un tratamiento de la información que haga factible la creación y el uso de indicadores pertinentes (5).

Debido a esta situación, en la Reunión de Redes y Campañas Regionales para América Latina y el Caribe celebrada en $\mathrm{La} \mathrm{Paz}^{3}$ se aprobó enviar una carta a los gobiernos latinoamericanos en la que se les solicita que renueven su compromiso con el Programa de Acción de la CIPD. La carta dice textualmente:

“. . . la última década ha sido el inicio de un camino de cambios en los países de América Latina y el Caribe. Hemos avanzado en la formulación de políticas, en la definición de planes y programas, en la definición de metas. Nuestros Estados han realizado, a la luz del Programa de Acción de El Cairo, cambios legislativos, cambios en la institucionalidad de los programas de salud en temas tan decisivos como adolescentes, VIH, acceso a anticoncepción, entre otros. El Cairo ha sido una carta de navegación para avanzar en forma integral en los temas de población y derechos sexuales y reproductivos. Pero, sin lugar a dudas, para que todos estos avances, desiguales en nuestra región, logren impactar en la vida y la situación de salud de las mujeres, de los hombres, de las y los adolescentes, es necesario que estos cambios y estos compromisos se adopten en forma ininterrumpida. Y aunque la formulación de las políticas desde esta perspectiva integral ha permitido poner a la población en el centro, desde el lugar de los derechos, los retos para el desarrollo y el empoderamiento nos siguen enfrentando como sociedad a dramas como el de la mortalidad materna, la feminización de la epidemia del sida, el incremento de la fecundidad adolescente no deseada, el cáncer de mama y de cuello uterino, este último, primera causa de mortalidad por cáncer entre mujeres en varios de nuestros países".

En el marco de la Reunión de La Paz, el Fondo de Población de las Naciones Unidas (FNUAP) solicitó a la Comisión Económica para América Latina y el Caribe (CEPAL) que emprendiera acciones conducentes a un acercamiento mayor de los países y territorios caribeños a los países latinoamericanos y propuso realizar una reunión de consenso de la subregión con la aspiración de que sus resultados pudieran utilizarse en otras partes de la Región de las Américas. 
En la Reunión Subregional de Evaluación del Programa de Acción de la Conferencia Internacional sobre la Población y el Desarrollo a 10 Años de su Adopción que se celebró los días 12 y 13 de noviembre de 2003 en Puerto España, Trinidad y Tabago, participaron representantes de 20 países y territorios del Caribe. Durante la reunión, Camille Robinson-Regis, Ministra de Planificación y Desarrollo del país sede, elogió los resultados de la CIPD y señaló que "las cuestiones de población ya no atañen meramente a cantidades, pues se trata de seres humanos vivos y activos que enfrentan problemas cotidianos. Se trata de la igualdad de género y el rechazo de la violencia por motivos de género en todas sus formas. Se trata de prepararse para tener un hijo deseado y ocuparse de que tenga una infancia saludable. Se trata de mejorar la calidad de la vida de toda la humanidad".

Este criterio fue apoyado por los participantes que resaltaron los avances alcanzados por los países del Caribe en la aplicación del Programa de Acción de la CIPD pese a los limitados recursos financieros disponibles.

En el informe Revisión de la Implementación del Programa de Acción de El Cairo en el Caribe (1994-2004): logros y dificultades, se dejó constancia de que el flujo de recursos para los países y territorios del Caribe es aún insuficiente y no se corresponde con las necesidades de la subregión. Por tal razón se convocó a hacer un uso más racional de los recursos existentes y a movilizar fondos adicionales para apoyar la formulación e implementación de programas y políticas de población y desarrollo en el Caribe. Los participantes agradecieron a la CEPAL y al UNFPA, entre otros, el apoyo brindado a la implementación del Programa de Acción de la CIPD y los invitaron a continuar su labor de coordinación, asistencia, cooperación y seguimiento de las actividades del Programa en el Caribe. De igual forma, se convocó a otras agencias de las Naciones Unidas, tales como la Organización Panamericana de la Salud y la Organización Mundial de la Salud, para apoyar la implementación del Programa en la subregión.

\section{Declaración del Caribe}

Un hito importante de la reunión subregional de Puerto España y del proceso de fortalecimiento de las políticas de género en el Caribe fue la aprobación de la Declaración del Caribe, en la cual se refrendan los retos y desafíos que deben servir de guía de acción para afianzar los logros alcanzados y avanzar en la consecución de las metas trazadas por la CIPD.

Entre los planteamientos fundamentales de la Declaración se pueden resaltar la necesidad de garantizar la vigencia de los derechos reproductivos y de la salud sexual y la salud reproductiva; la igual- dad de género y la ampliación de los medios de acción de la mujer; el reconocimiento de los derechos de los adolescentes y los jóvenes a tener información, educación y acceso a servicios de salud reproductiva; y la necesidad de combatir la pandemia del sida y la infección por VIH en el Caribe, segunda región del mundo más fuertemente golpeada por la enfermedad. Además, se expone la necesidad de adoptar medidas de prevención, tratamiento, atención e información, sustentadas por políticas que no excluyan a nadie por su clase, origen étnico, género, color de la piel u orientación sexual.

En la Declaración del Caribe también se reflejó el daño que han causado a los pueblos, economías y sociedades de los países caribeños los programas de ajuste estructural, la pesada carga de la deuda externa y la falta de equidad en las prácticas comerciales. Se hizo un llamado a la comunidad internacional para fomentar y adoptar un sistema internacional de comercio equitativo, seguro y no discriminatorio. Consecuentemente, se hizo un llamado a los países y territorios de la subregión para que aumentaran la colaboración en aras de mejorar el bienestar social y económico de la población.

Los países participantes hicieron explícito en la Declaración su compromiso de continuar los procesos nacionales de reforma legislativa y esforzarse en su implementación eficaz, para garantizar el total cumplimiento del Programa de Acción de la CIPD y el Plan de Acción consensuado para el Caribe.

Otro elemento importante que quedó plasmado en la Declaración fue el referido a las necesidades de información de la subregión. Se convocó a los países a establecer programas nacionales y subregionales de capacitación a fin de aprovechar mejor las nuevas tecnologías, de mejorar la recolección, procesamiento y análisis de la información, de garantizar la diseminación de información oportuna y desagregada por sexo, y de garantizar que a todos los niveles se tomen decisiones basadas en pruebas.

En la Declaración se dejó constancia de la gravedad de algunos problemas que afectan a la subregión, como el contrabando y tráfico de personas, especialmente de mujeres y niños; el "robo de cerebros"; el retorno de los emigrantes de uno u otro sexo y las deportaciones; las fronteras de seguridad establecidas a raíz de los trágicos acontecimientos del 11 de septiembre de 2001 en los Estados Unidos de América; y la libertad de movimiento de las personas bajo las disposiciones de un mercado común del Caribe. En el documento aprobado se llama a establecer compromisos concretos para atender y dar respuesta a esos importantes problemas.

Los países firmantes reconocieron también el apoyo financiero brindado por organizaciones no gubernamentales (ONG), el sector privado y otros donantes con vistas a garantizar la asistencia técnica requerida para implementar el Programa de Acción de 
la CIPD en el Caribe y se reiteró la necesidad de un mayor financiamiento para desarrollar las acciones propuestas. En particular, se resaltó el importante papel desempeñado por las ONG en la elaboración de políticas de desarrollo y en la implementación y conducción de servicios de promoción de los derechos reproductivos y de salud sexual y salud reproductiva, por lo cual se hizo un llamado a los países de la subregión para favorecer las acciones conjuntas con las ONG. La experiencia ha demostrado que el trabajo de las ONG, así como de las redes sociales, favorece la sustentabilidad de los procesos de implementación de programas y acciones en el ámbito de los derechos y de la salud sexual y reproductiva.

\section{Conclusiones}

El Programa de Acción de la CIPD, aprobado hace 10 años, ha estimulado a los Gobiernos de los países y territorios del Caribe a implementar los acuerdos establecidos en esa conferencia, lo que ha llevado a concertar alianzas, aunar voluntades y esfuerzos, movilizar recursos y promover "lentos pero acertados" cambios culturales, jurídicos y normativos en el complejo ámbito de la salud sexual y reproductiva. En la subregión del Caribe también se han logrado avances, aunque aún resultan insuficientes. Se debe continuar creando condiciones y oportunidades para promover el desarrollo de la autonomía, el empoderamiento y le equidad de clase, etnia y género en materia de población y desarrollo, y en el ámbito de los derechos reproductivos y de la salud sexual y reproductiva.

La Declaración del Caribe constituye una guía para la acción. Falta echarla a andar y en eso debemos participar todos, para poder encontrarnos dentro de cinco años, en el próximo balance de los acuerdos de la CIPD, con menos restricciones y mayores avances en el camino de mejorar la salud y el bienestar de la población caribeña, que bien lo merece.

\section{SYNOPSIS \\ Challenges facing Caribbean countries 10 years after the International Conference on Population and Development in Cairo, Egypt}

At the International Conference on Population and Development (ICPD) that was held in Cairo, Egypt, in 1994, participants acknowledged that population, economic growth, and sustainable development are concepts that are closely linked, and important strides were made in terms of increased recognition of sexual and reproductive rights. The Programme of Action ratified at that Conference was adopted as a platform for designing national and international policies in the areas of population and development for a period of twenty years. However, in Latin America and the Caribbean all types of obstacles-financial, institutional, and human-still stand in the way of attaining the goals of the Programme of Action, and some governments have established measures that undermine their people's exercise of sexual and reproductive rights. The Caribbean Subregional Meeting to Assess the Implementation of the Programme of Action of the International Conference on Population and Development 10 Years after its Adoption was held in Port of Spain, Trinidad and Tobago, in November of 2003. At the meeting, which was attended by representatives from 20 Caribbean countries and territories, a call was made for more rational use of available resources and for mobilization of additional funds for developing and implementing population and development programs and policies in the Caribbean. The meeting also saw the approval of the Caribbean Declaration, which lays out the challenges that should serve as the roadmap for taking actions to consolidate the progress achieved so far and come closer to attaining the goals established by the ICPD. In the Declaration, the countries and territories of the Caribbean asserted their commitment to continue legislative reforms at the national level while seeking to enforce these reforms in an effort to ensure implementation of the ICPD's Programme of Action and of the Caribbean Plan of Action for Population and Development that was adopted in 1996 by the Economic Community for Latin America and the Caribbean.

\section{REFERENCIAS}

1. Fondo de Población de las Naciones Unidas. Reporte Final. Reunión Consultiva entre el Fondo de Naciones Unidas (UNFPA) y ONGs sobre la Implementación del Programa de Acción de la CIPD en América Latina y el Caribe. Nueva York, mayo de 2003.

2. Fondo de Población de las Naciones Unidas. Informe. Conferencia Internacional sobre la Población y el Desarrollo, El Cairo, Egipto, septiembre de 1994. Caracas, Venezuela: UNFPA; 2003.
3. Centro Latinoamericano de Perinatología y Desarrollo Humano. Información en salud materna y perinatal (América Latina y Caribe) [sitio en Internet]. Hallado en: http://www.paho.org/Spanish/ CLAP/01index.htm. Acceso el 24 de diciembre de 2003.

4. Artiles L. Políticas públicas y equidad de género. Centroamérica y Caribe. XII Congress of the International Association of Health Policy. XIV Jornadas de Debate de la Federación de Asociaciones para la De- fensa de la Sanidad Pública. Palma de Mallorca, España, 21 al 24 de mayo de 2002.

5. Gómez Gómez E. La salud y las mujeres en América Latina y el Caribe: viejos problemas y nuevos enfoques. Santiago, Chile: Comisión Económica para América Latina y el Caribe; 1997. (Serie Mujer y Desarrollo. Unidad Mujer y Desarrollo No. 17). 